\title{
SCPH 2015 : Tout le monde y trouve son compte
}

\author{
par Neil J MacKinnon
}

L 'une des façons les plus concrètes pour que les pharmaciens d'hôpitaux de tout le Canada travaillent ensemble à l'amélioration du système de distribution des médicaments est le projet SCPH 2015, un programme visant l'excellence dans la pratique pharmaceutique pour les pharmaciens ouvrant dans les établissements de santé (www.cshp.ca/programs/cshp2015/ index_e.asp). Le dessein général du projet SCPH 2015 est d'accroître l'utilisation efficace, scientifique et sûre des médicaments ainsi que de contribuer notablement à la santé publique, et du coup, d'améliorer la santé des Canadiens. La réussite sera au rendez-vous lorsque seront atteints les six buts assortis des 36 objectifs mesurables. Le projet SCPH 2015 peut aider à faire évoluer la pharmacie d'hôpital au Canada grâce à un élan concerté et décisif, en mettant ainsi les pharmaciens dans une position où ils peuvent exercer de façon proactive une influence favorable sur les soins aux patients.

À vrai dire, tout le monde y trouve son compte avec le projet SCPH 2015. "Comment? », vous demandez-vous? Je suis heureux que vous vous posiez la question. Permettez-moi de vous le montrer.

Si vous êtes un pharmacien clinicien, le projet SCPH 2015 peut vous aider à faire la promotion de l'excellence de la pharmacie dans le milieu où vous exercez. Le premier but du projet SCPH 2015 est d'accroître le degré d'intervention des pharmaciens auprès de chaque patient hospitalisé afin d'assurer l'utilisation optimale des médicaments. Les membres de la Société canadienne des pharmaciens d'hôpitaux peuvent se prévaloir d'une trousse qui s'intéresse à la prestation de soins complexes aux patients hospitalisés et au rôle du pharmacien dans la prestation de ces soins. La deuxième trousse, "From Paper to Practice: Incorporating Evidence into Your Pharmacy Practice", contient du matériel de soutien qui favorise une pratique de la pharmacie fondée sur des données probantes et vise l'atteinte du troisième but.
Si vous êtes un pharmacien ayant des responsabilités en matière de distribution de médicaments, le projet SCPH 2015 peut vous aider à optimiser le système de distribution des médicaments dans votre établissement. Un autre des buts du projet SCPH 2015 est d'accroître le rôle joué par les services de pharmacie des établissements de santé dans l'amélioration de l'utilisation sécuritaire des médicaments. Une autre trousse bientôt disponible, "One dose at a time: Implementing a unit-dose medication management system ", vous proposera des conseils pratiques pour améliorer votre système de distribution des médicaments.

Si vous avez des fonctions de supervision, de gestion ou de direction d'un service de pharmacie, vous pouvez utiliser le projet SCPH 2015 pour justifier et appuyer de nouveaux services ou programmes pharmaceutiques et pour l'intégrer dans le plan stratégique de votre service pour ce qui est de l'excellence des soins et de la pratique pharmaceutiques. Vous pouvez juger des progrès par rapport à ses buts dans une section spéciale sur le projet SCPH 2015 du Sondage bisannuel sur les pharmacies hospitalières canadiennes. De plus, un tableau de concordance établit le lien entre les 36 objectifs du projet SCPH 2015 et d'autres initiatives connexes (notamment, «Des soins de santé plus sécuritaires maintenant! " et Agrément Canada) et fournit les données scientifiques à l'appui de chacun de ces objectifs. Le sondage bisannuel sur le projet SCPH 2015 auprès des établissements de santé évalue à la fois le classement des priorités et le degré d'atteinte des buts et objectifs.

En dernier lieu, si vous êtes un patient, vous serez rassuré de savoir que le projet SCPH 2015 contribuera à veiller à l'utilisation efficace, scientifique et sûre des médicaments dans les établissements de santé partout au Canada.

Neil J MacKinnon, B. Sc. (Pharm.), M. Sc. (Pharm.), Ph. D., FCSHP, est président sortant et agent de liaison pour la vision de la SCPH. 